\title{
The construction of exact multipolar equilibria of the two-dimensional Euler equations
}

\author{
Darren Crowdy ${ }^{\mathrm{a})}$ \\ Department of Mathematics, Imperial College of Science, Technology and Medicine, \\ 180 Queen's Gate, London SW7 2BZ, United Kingdom
}

(Received 12 April 2000; accepted 10 September 2001)

\begin{abstract}
Using ideas involving the Schwarz function of analytic curves, a new class of exact multipolar equilibria of the two-dimensional Euler equations characterized by an annular region of vorticity enclosing a region of irrotational fluid is constructed. The results generalize a recently derived class of exact solutions for multipolar vortex equilibria [Crowdy, Phys. Fluids 11, 2556 (1999)]. The solutions have many qualitative similarities to the multiple-vortex nonlinear saturation states of an unstable annulus of uniform vorticity. More generally, the results suggest the possibility of constructing multipolar equilibria of the steady Euler equations having distributed vortical regions of more or less arbitrary geometrical complexity. (c) 2002 American Institute of Physics.
\end{abstract}

[DOI: $10.1063 / 1.1420746]$

\section{INTRODUCTION}

Coherent vortical structures are now known to constitute an important feature of many two-dimensional and quasigeostrophic flows. ${ }^{1}$ They arise in many aspects of astrophysical, geophysical, and meteorological fluid dynamics. In an initial state of randomly distributed vorticity, for example, the cascade of energy to larger scales is responsible for the formation of such vortical structures. ${ }^{2}$ While monopoles and dipoles are the most ubiquitous structures (characterized by one and two vorticity maxima respectively), laboratory experiments $^{3-6}$ and numerical simulations ${ }^{7-11}$ have shown that higher order structures, such as tripoles and quadrupoles $^{5,6,11}$ (as well as even higher order structures), arise from the instability of isolated circular vortices (usually with zero total circulation). A tripole is characterized by three vorticity maxima- a central core region with vorticity of one sign surrounded by two satellite vortices both of opposite sign. A tripolar-like vortex has been observed in real physical flows ${ }^{12}$ and the possibility of its emergence from an unstable monopolar vortex was originally mentioned by Leith. ${ }^{13}$ A quadrupole has a central core surrounded by a triangular array of three such satellites. The general class of vortices of this general kind have been dubbed "multipolar vortices" and the formation, structure, and stability properties of such vortices is a topic of much recent research activity. Typically, such vortex structures rotate at a constant angular velocity. We refer the reader to Ref. 6 for a detailed discussion of the general properties of multipolar vortices.

Owing to the complicated structure of these multipolar vortices, most investigations of them have involved either laboratory experiments or full numerical simulations, although simple point vortex models can often capture many aspects of realistic flow situations (e.g., Ref. 14). It is clearly

a)Telephone: (020) 7594 8587; fax: (020) 7594 8517; electronic mail: d.crowdy@ic.ac.uk of interest, from a theoretical point of view, to find effective models of such vortices, or ideally, some mathematical solutions of the Euler equations which resemble multipolar vortices and which can be studied explicitly. In this vein, Kloosterziel and Carnevale ${ }^{15}$ have recently examined the possibility of approximating the evolutionary dynamics between these equilibria by low-order dynamical systems while, with a view to extending our theoretical understanding of these structures as mathematical solutions of the Euler equations, the present author recently pointed out ${ }^{16}$ that there exists a class of exact solutions to the steady twodimensional Euler equations which share all the qualitative properties of multipolar vortices observed in practice (physical observation, experiments, and numerical simulations). The new solutions are finite-area patches of nonzero vorticity and therefore, unlike simple point-vortex models, provide insight into the shapes of vortical equilibria of the Euler equation.

It was pointed out in Ref. 16 that the solutions derived there had the intriguing property of being "invisible" in that they do not induce any irrotational velocity field outside the support of the vorticity. Such vortices therefore only interact when the overlap. This prompts a very natural question which forms the basis of this paper: what happens when such vortices do overlap? In particular, can overlapping of such vortices produce more coherent vortex equilibria of the $2 \mathrm{D}$ Euler equations, perhaps with vortical regions having even more complex shapes? This paper answers this question and shows that, in certain circumstances, it is possible for such vortices to merge in such a way as to form a steady higher order vortical structure. Indeed, our principal result is to show that, within the class of solutions under consideration, while (shielded) monopoles cannot combine in pairs to form compound equilibria, higher-order equilibria can be formed provided at least three monopoles combine in an annular configuration thereby forming a vortical region of higher connectivity (in fact, doubly connected). In the process, we 
essentially produce a broad new class of exact multipolar solutions of the steady two-dimensional Euler equations. This paper constitutes a natural sequel to Ref. 16 .

Many investigators have considered what happens when two or more coherent structures interact. Saffman and Szeto $^{17}$ showed that two patches of like-signed uniform vorticity (i.e., V-states) cannot get too close together and continue to exist in a co-rotating equilibrium, while Pierrehumbert ${ }^{18}$ considered the case of two opposite-signed symmetrical vortices. Dritschel ${ }^{19}$ later generalized the Saffman-Szeto scenario to the case when an array of $k$ likesigned uniform vortex patches exist in an annular co-rotating equilibrium. Dritschel suggested that such equilibria might be the nonlinear saturation states of an unstable annulus of

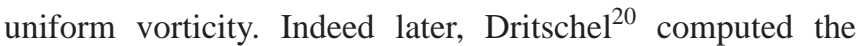
nonlinear evolution of an annulus of uniform vorticity and showed that, under suitable circumstances, it did indeed destabilize into an annular array of distinct vorticity maxima which looked very similar to the 5-patch annular configuration computed in Ref. 19. The new equilibrium solutions to be presented here have very strong similarities to the numerical solutions of annular arrays of co-rotating V-states discussed by Dritschel. ${ }^{19,20}$ Indeed, we believe our new solutions to be a mathematically exact subclass of this general class of equilibria of the 2D Euler equation.

\section{OVERLAPPING MONOPOLAR VORTICES}

Saffman and Szeto ${ }^{17}$ explain the nonexistence of a corotating equilibrium when two like-signed vortices draw too close together as being due to the strain field induced by one vortex being too strong for the other vortex to exist as a separate entity. In the case of the solutions of Ref. 16 which induce no straining flow outside the support of the vorticity, this mechanism no longer represents a possible reason for the nonexistence of steady solutions. We therefore suggest that such vortices might be able to draw arbitrarily close together and "merge" to form higher-order compound vortical structures.

To illustrate the idea, consider the exact solution of the Euler equations referred to in Ref. 16 as a shielded Rankine vortex given, in cylindrical polar coordinates $(r, \theta)$, by the velocity field $\mathbf{u}=(0, V(r))$ where the azimuthal velocity field is given by

$$
V(r)=\left\{\begin{array}{l}
\frac{\omega_{0} r}{2}-\frac{\omega_{0}}{2 r}, \quad r \leqslant 1, \\
0, \quad r>1,
\end{array}\right.
$$

and where $\omega_{0}$ is the constant uniform vorticity inside the circular patch. This solution might equally well be referred to as a "shielded line vortex" because the patch of uniform vorticity surrounding the central line vortex can be viewed as shielding it. Here, however, we continue to employ the name introduced in Ref. 16. This vortex will be a key building block in the development. It is from understanding this vortex from the point of view of the Schwarz function of the circle $r=1$ that forms the basis of the derivation of the higher-order multipolar structures of Ref. 16.
By definition, the Schwarz function of a general closed analytic curve $\partial D$ surrounding a bounded, simply connected domain $D$ in the plane is the (unique) function, analytic in an annular domain containing the curve $\partial D$, satisfying the relation

$$
S(z)=\bar{z}, \quad \text { everywhere on } \partial D .
$$

In the case in which $\partial D$ is the circle defined by $z \bar{z}=1$ it is clear that everywhere on $\partial D$,

$$
\bar{z}=\frac{1}{z} \text {. }
$$

The function of $z$ on the right hand side is analytic in, for example, the annular domain $0.9<|z|<1.1$ which contains the unit circle $|z|=1$. The function also satisfies Eq. (2). We therefore recognize it as the Schwarz function of the circle, i.e.,

$$
S(z)=\frac{1}{z} .
$$

The following analysis will be self-contained in that all results regarding the Schwarz functions needed for the present application will be cited here. For more details, however, the interested reader is referred to a monograph by Davis. ${ }^{21}$ Now consider the velocity field Eq. (1) rewritten in terms of $z$ $=x+i y$ and its complex conjugate. Using the fact that the radial and tangential components of a general twodimensional incompressible velocity field $(U, V)$ is related to the streamfunction by the formula

$$
2 i \psi_{z}=e^{i \theta}(U-i V),
$$

it can be deduced that the streamfunction associated with Eq. (1) is

$$
\psi=-\frac{\omega_{0}}{4}\left(z \bar{z}-\int \frac{z}{z^{\prime}} d z^{\prime}-\int^{\bar{z}} \frac{1}{z^{\prime}} d z^{\prime}\right) .
$$

Using the identification Eq. (4) this is equivalent to

$$
\psi=-\frac{\omega_{0}}{4}\left(z \bar{z}-\int^{z} S\left(z^{\prime}\right) d z^{\prime}-\int^{\bar{z}} \bar{S}\left(z^{\prime}\right) d z^{\prime}\right),
$$

where the conjugate function $\bar{S}(z)$ is defined by $\overline{S(\bar{z})}$. Formula (7) provides the key route to generalization developed in Ref. 16.

The velocity field everywhere outside the vortex in Eq. (1) is quiescent. The same is true of all the generalized solutions found in Ref. 16 where the adjective "invisible" is used to describe such vortices. Consider two shielded Rankine vortices sitting close together but not touching. Such a configuration also constitutes a self-consistent global solution of the steady Euler equations precisely because the vortices do not interact. Indeed, two such vortices can draw so close together that they actually touch. This is possible because neither vortex induces any strain field outside the support of its own vorticity. It is natural to ask whether the two such monopolar solutions can merge, not in a dynamical sense, but in the sense that the class of equilibria can be continued via a continuous sequence of steady solutions 
which form higher-order steady multipolar structures within the same class of solutions considered in Ref. 16, i.e., nonrotating and surrounded by quiescent fluid.

There is a further reason to suspect that this idea of steady merger might be feasible. At a point where two shielded Rankine vortices touch, the resulting configuration will have a cusp singularity in its boundary at the point of contact. The vorticity in the neighborhood of this cusp will be uniform. Overman ${ }^{22}$ has made a careful study of "limiting V-states" (i.e., limiting shapes of patches of uniform vorticity) and shown, by means of local arguments in the neighborhood of a point of nonanalyticity in the boundary of a uniform $\mathrm{V}$-state, that the only possible limiting states possess either $90^{\circ}$ corners or cusps. Overman's analysis is therefore consistent with, and does not immediately rule out, the possibility of continuously smoothing out a cusped point of contact as two initially disjoint vortex structures come together.

This question of the steady merger of the invisible vortices of Ref. 16 is not a trivial one. Indeed, the question just posed of whether just two shielded Rankine vortices can merge to form a higher-order, shielded, dipolar structure (within the same class of solutions) characterized by two localized vorticity maxima has a negative answer. This question was answered implicitly in Ref. 16 where it is found that there does not exist a simply connected dipolar vortical patch surrounded by quiescent fluid. Thus while it is possible for two shielded Rankine vortices to approach eachother so closely that they touch, it is not in fact possible to continue these solutions so that the monopoles "overlap" and remain in equilibrium.

\section{ANNULAR CONFIGURATIONS OF VORTICES}

Given this observation, it might appear that the proposed search for compound vortices formed by the overlapping of the canonical monopolar vortices Eq. (1) is destined for failure. However, many studies reveal that vortices typically arrange themselves so that they consist of a finite array of vorticity maxima congregating in a co-rotating annular configuration. The classic studies of a co-rotating ring of line vortices by Thomson (and others) are well-known. ${ }^{23}$ It is also known that there exist annular configurations of vorticity maxima associated with regions of distributed vorticity with central core regions consisting wholly of irrotational fluid. ${ }^{19,20}$ Also, in a typical multipolar vortex (e.g., Ref. 11), $k$ satellite vortices congregate in an annular configuration and enclose a core region consisting of a region of vorticity of opposite sign usually also possessing some enclosed regions of irrotational fluid.

Motivated by this, we explore the situation in which a collection of $k$ shielded Rankine vortices come together in such a way that they form an annular (i.e., doubly connected) region of vorticity enclosing a finite region of irrotational fluid and surrounded by irrotational fluid. It will be shown that such solutions exist and, moreover, that they are precisely a result of the merging of an annular array of the canonical monopolar vortices Eq. (1). The solutions will be found by generalizing the methods presented in Ref. 16. Be-

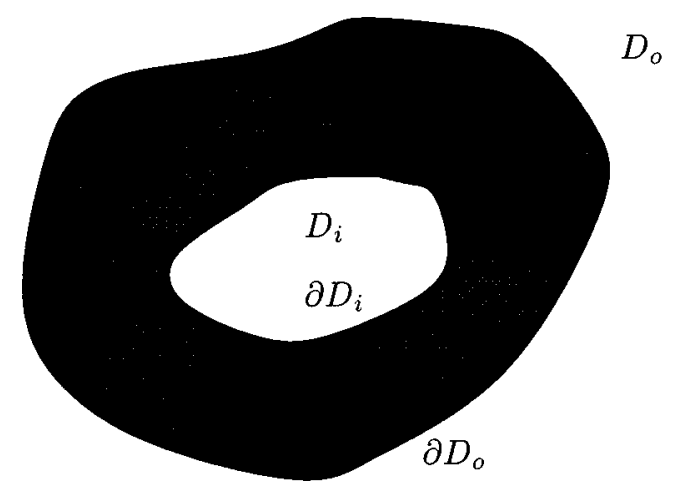

FIG. 1. Annular patch of vorticity $D$ (shaded): the interior and exterior regions of irrotational fluid are denoted $D_{i}$ and $D_{0}$, respectively. The interior boundary of $D$ is denoted $\partial D_{i}$, the exterior boundary denoted $\partial D_{0}$.

cause the support of the vorticity in the resulting compound structure is now doubly connected, the analysis of Ref. 16 must be generalized in a nontrivial way.

We review and summarize the results of Ref. 16. This work pointed out the relevance to multipolar vortex solutions of the steady Euler equations of a streamfunction defined by $\psi(z, \bar{z})$

$$
=\left\{\begin{array}{l}
-\frac{\omega}{4}\left(z \bar{z}-\int^{z} S\left(z^{\prime}\right) d z^{\prime}-\int^{\bar{z}} \bar{S}\left(z^{\prime}\right) d z^{\prime}\right), \quad \text { in } D, \\
0, \text { outside } D,
\end{array}\right.
$$

where $S(z)$ is the Schwarz function of the boundary of a finite-area, simply connected patch of nonzero vorticity. The function Eq. (8) also has relevance in other quite different (more mathematical) contexts and is sometimes referred to as a modified Schwarz potential. ${ }^{24}$

As pointed out in Ref. 16, the special choice of streamfunction Eq. (8) is remarkable in that it simultaneously satisfies both the kinematic and dynamic boundary conditions on the boundary of $D$ (i.e., the boundary of the region of nonzero vorticity). If one then restricts the class of vortex patches to those which have a Schwarz function which is meromorphic inside the patch with just simple pole singularities having real residues, it was discovered that one can apply the non-self-induction hypothesis to obtain exact, selfconsistent solutions of the steady Euler equations. We refer the reader to Ref. 16 for more details.

Now consider such a patch with an additional interior region of enclosed irrotational fluid denoted $D_{i}$. The region of irrotational fluid outside the vortex patch will be denoted $D_{0}$. For convenience, we will refer to the outer boundary (between $D$ and $D_{0}$ ) as $\partial D_{0}$ and the inner boundary (between $D$ and $\left.D_{i}\right)$ as $\partial D_{i}$. Figure 1 provides a schematic. The difficulty of generalizing Eq. (8) to an annular (i.e., doubly connected) region of vorticity is immediately seen when one considers the fact that such an annular region of vorticity $D$ now has two disjoint bounding curves. In general, both $\partial D_{0}$ and $\partial D_{i}$, being disjoint closed analytic curves, will each define an associated Schwarz function denoted $S_{0}(z)$ and $S_{i}(z)$, respectively, i.e., 


$$
\begin{aligned}
& \bar{z}=S_{0}(z), \quad \text { on } \partial D_{0}, \\
& \bar{z}=S_{i}(z), \quad \text { on } \partial D_{i} .
\end{aligned}
$$

For general annular domains, these Schwarz functions are different. It is therefore not immediately clear how to generalize the streamfunction $\psi(z, \bar{z})$ as given in Eq. (8): following the analysis in Ref. 16 a streamfunction defined by

$$
\psi(z, \bar{z})=-\frac{\omega}{4}\left[z \bar{z}-\int{ }^{z} S_{0}\left(z^{\prime}\right) d z^{\prime}-\int{ }^{\bar{z}} \bar{S}_{0}\left(z^{\prime}\right) d z^{\prime}\right]
$$

will satisfy both the kinematic and dynamic boundary conditions on the exterior boundary $\partial D_{0}$ provided there is quiescent fluid outside the patch, while a streamfunction defined by

$$
\psi(z, \bar{z})=-\frac{\omega}{4}\left[z \bar{z}-\int^{z} S_{i}\left(z^{\prime}\right) d z^{\prime}-\int \bar{z}^{\bar{z}} \bar{S}_{i}\left(z^{\prime}\right) d z^{\prime}\right]
$$

will satisfy the kinematic and dynamic boundary conditions on the interior boundary $\partial D_{i}$ provided there is quiescent fluid in the region enclosed by the annular vortical region. But, between the two boundaries (i.e., the vortical region) it is necessary to define a common streamfunction. For general classes of annular vortical domains, Eqs. (10) and (11) will define different streamfunctions.

The key to generalization is to restrict to a special class of doubly connected vortical domains. We now suppose it is possible to find a doubly connected domain $D$ which has the distinguished property that

$$
S_{0}(z)=S_{i}(z) \equiv S(z), \quad \text { in } D,
$$

except at a finite number $k$ of simple pole singularities $\left\{z_{j} \mid j=1, \ldots, k\right\}$ of $S(z)$ which are strictly inside $D$. In other words, we consider a special class of domains in which both the outer and inner bounding curves of the annular domain have the same Schwarz function which continues meromorphically throughout the vortex patch. In addition, this (common) Schwarz function [i.e., $S(z)$ ] will be assumed to have only simple pole singularities $\left\{z_{j} \mid j=1, . ., k\right\}$ in $D$ each having purely real residues.

It has not yet been established that such special domains exist, however, we now show why such domains are of interest to us. Consider the streamfunction defined by

$$
\psi(z, \bar{z})
$$

$$
=\left\{\begin{array}{l}
0, \quad \text { in } D_{0} \\
-\frac{\omega}{4}\left(z \bar{z}-\int{ }^{z} S\left(z^{\prime}\right) d z^{\prime}-\int^{\bar{z}} \bar{S}\left(z^{\prime}\right) d z^{\prime}\right), \text { in } D, \\
0, \text { in } D_{i}
\end{array}\right.
$$

where $S(z)$ is defined by Eq. (12). The vorticity associated with this streamfunction is equal to a uniform $\omega$ everywhere inside $D$ except possibly at any singularities of $S(z)$. Inside $D$, the total derivative $d \psi$ is given by

$$
\begin{aligned}
d \psi & =\psi_{z} d z+\psi_{\bar{z}} d \bar{z}, \\
& =-\frac{\omega}{4}(\bar{z}-S(z)) d z-\frac{\omega}{4}(z-\bar{S}(\bar{z})) d \bar{z}
\end{aligned}
$$

On the boundary $\partial D_{0}$, Eqs. (9) and (12) imply that $d \psi=0$ so that $\partial D_{0}$ is a streamline. Therefore, the kinematic boundary condition is satisfied on this boundary. Moreover, $u$ $-i v=2 i \psi_{z}=0$ on $\partial D_{0}$ so that velocities are continuous on the outer boundary. This implies that fluid pressure is continuous on $\partial D_{0},{ }^{25}$ and therefore that the dynamic boundary condition is satisfied. Because of stipulation Eq. (12), exactly the same arguments hold on the inner boundary $\partial D_{i}$. If $S(z)$ is assumed to have simple pole singularities in $D$ with real residues, then the streamfunction Eq. (13) will have line vortex singularities at these points. For a consistent solution of the steady Euler equations it is necessary, by the Helmholtz vortex theorems, ${ }^{25}$ to ensure that all such line vortices are stationary under the effects of the local non-self-induced velocity field. This important physical constraint is discussed further in Sec. V once we have found a way to construct the special class of doubly connected domains now of interest to us (these domains are related to the theory of quadrature domains $\left.{ }^{24,26}\right)$.

\section{CONFORMAL MAPPING}

As in Ref. 16, the most convenient way to parametrize the class of domains now under consideration is to introduce a conformal map $z(\zeta)$ from a canonical doubly connected region. By Riemann's theorem, ${ }^{27}$ for any doubly connected region there exists a conformal map from an annulus $\rho<|\zeta|<1$ for some choice of $\rho$. This parameter $\rho$ (known as the conformal modulus of the mapping-see Ref. 27) must be determined as part of the solution. The circle $|\zeta|=1$ will map to the exterior boundary of the patch $\partial D_{0}$, while $|\zeta|=\rho$ will map to the interior boundary $\partial D_{i}$. See Fig. 2 for a schematic.

It is convenient to write the function $S(z)$ in terms of the parametric $\zeta$-variable which, henceforth, will be used (along with its conjugate variable) to parametrize all physical quantities associated with the flow. On $|\zeta|=1$, the Schwarz function $S_{0}(z)$ is defined by

$$
S_{0}(z)=\bar{z}
$$

Using the fact that $\bar{\zeta}=\zeta^{-1}$ on $|\zeta|=1$ it follows that $S_{0}(z)$ can be written

$$
S_{0}(z(\zeta))=\overline{z(\zeta)}=\bar{z}\left(\zeta^{-1}\right), \quad \text { on }|\zeta|=1 .
$$

Here $\bar{z}(\zeta)$ denotes the conjugate function to $z(\zeta)$ defined via

$$
\bar{z}(\zeta)=\overline{z(\bar{\zeta})}
$$

By analytic continuation, Eq. (16) also holds off the circle $|\zeta|=1$. Using the fact that $\bar{\zeta}=\rho^{2} \zeta^{-1}$ on $|\zeta|=\rho$ the Schwarz function $S_{i}(z)$ can similarly be written as

$$
S_{i}(z(\zeta))=\overline{z(\zeta)}=\bar{z}\left(\rho^{2} \zeta^{-1}\right),
$$




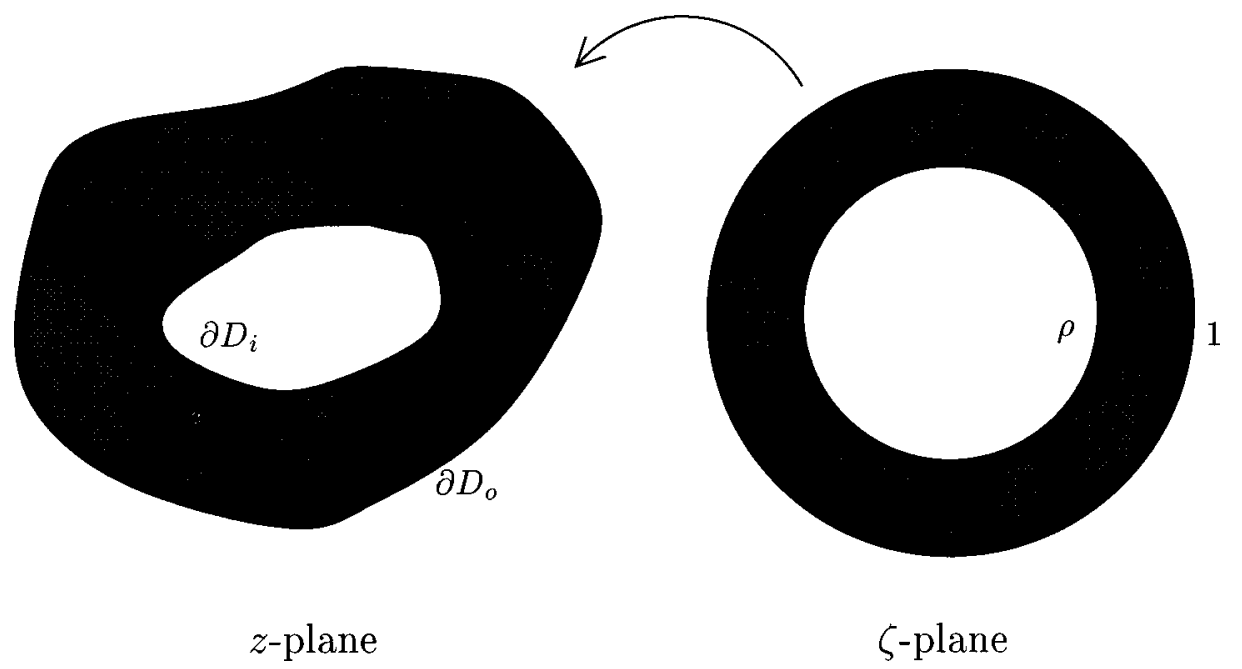

FIG. 2. Conformal mapping domains.

which similarly also holds off the circle $|\zeta|=\rho$ by analytic continuation. Therefore, comparing Eqs. (16) and (18), in order that Eq. (12) should hold, the conformal map must satisfy the functional relation

$$
\bar{z}\left(\zeta^{-1}\right)=\bar{z}\left(\rho^{2} \zeta^{-1}\right),
$$

for all $\zeta \neq 0$. Functions satisfying such a multiplicative periodicity property are studied in classical texts on function theory (e.g., Ref. 28) but all results needed for the present application will be explicitly stated below.

In order to explicitly construct functions satisfying Eq. (19) it is necessary to introduce a single special function. Consider the function $P_{k}(\zeta)$, indexed by a nonzero positive integer $k$, defined by the following infinite-product expansion:

$$
P_{k}(\zeta) \equiv\left(1-\zeta^{k}\right) \prod_{n=1}^{\infty}\left(1-\rho^{2 n k} \zeta^{k}\right)\left(1-\rho^{2 n k} \zeta^{-k}\right) .
$$

This function has some special properties which will enable us to construct a conformal mapping satisfying Eq. (19). First, observe that some simple direct manipulations of the infinite-product definitions reveal that $P_{k}(\zeta)$ has the following transformation properties:

$$
P_{k}\left(\zeta^{-1}\right)=P_{k}\left(\rho^{2} \zeta\right)=-\frac{1}{\zeta^{k}} P_{k}(\zeta)
$$

We now pick a positive integer $k$ and define the following conformal map using the special function $P_{k}(\zeta)$ :

$$
z(\zeta)=R \zeta \frac{P_{k}\left(\zeta \eta_{1}^{-1}\right)}{P_{k}\left(\zeta \zeta_{1}^{-1}\right)}
$$

where $R$ is a real parameter and the real parameters $\rho, \eta_{1}$, and $\zeta_{1}$ are related via

$$
\frac{\rho^{2} \eta_{1}^{k}}{\zeta_{1}^{k}}=1
$$

Given this relation, the conformal map Eq. (22) contains three independent (real) parameters, i.e., $\rho, \zeta_{1}$, and $R$. Using Eq. (21), routine algebraic manipulation reveals that for any integer $k$, the conformal map Eq. (22) with parameters satisfying Eq. (23) exactly satisfies the functional equation (19).

It is important to observe that if $z(\zeta)$ is known in the annulus $\rho<|\zeta|<\rho^{-1}$, then Eq. (19) provides the analytic continuation of $z(\zeta)$ into every other annulus $\rho^{2 j+1}<|\zeta|$ $<\rho^{2 j-1}$ where $j$ is any integer $(j \neq 0)$. We therefore refer to the annulus

$$
\rho<|\zeta|<\rho^{-1}
$$

as the fundamental annulus. The analyticity/singularity structure of $z(\zeta)$ in all other annuli is equivalent to the analyticity structure in this fundamental annulus. Note also that $z(\zeta)$ cannot have singularities in the sub-annulus $\rho<|\zeta|<1$ of the fundamental annulus because this corresponds to the preimage of the mapped vortical region. It is therefore enough the consider the singularities of $z(\zeta)$ in the single annulus $1<|\zeta|<\rho^{-1}$.

Because $P_{k}(\zeta)$ is just a function of $\zeta^{k}$, it is easy to see that, for any choice of integer $k, z(\zeta)$ as defined in Eq. (22) is invariant under the transformation $\zeta \mapsto \zeta \omega_{k}^{j}$, where $\omega_{k}$ $=e^{2 \pi i / k}$ and $j$ is any integer. Thus, the conformal map Eq. (22) represents a mapping to a vortical region possessing a $k$-fold rotational symmetry. Moreover, if we choose all the parameters in Eq. (22) to be real, then the annular patch of vorticity will be symmetric with respect to reflections in the $x$-axis. In particular, this means that the conjugate function $\bar{z}(\zeta)$ is equal to $z(\zeta)$, i.e.,

$$
\bar{z}(\zeta)=z(\zeta) .
$$

We will restrict consideration to this class of vortex patches.

If $\zeta_{1}$ is chosen to be in the real interval $1<\zeta<\rho^{-1}$ (recall that, according to the reasoning above, it is enough to consider the singularity structure of $z(\zeta)$ in this particular annulus alone), then the conformal map $z(\zeta)$ will have $k$ symmetrically placed simple pole singularities at points $\zeta$ $=\zeta_{1} \omega_{k}^{j}, j=0,1,2, \ldots, k-1$. This implies, from Eq. (16), that $S(z(\zeta))$ will have simple pole singularities inside the vortical region at points corresponding to $\zeta_{1}^{-1} \omega_{k}^{j}, j=0,1,2, \ldots$, $k-1$. From the streamfunction Eq. (13), it is clear that physically this corresponds to the presence of $k$ line vortex singu- 
larities at symmetrically distributed points inside an otherwise uniform annular patch of vorticity. In order for the streamfunction Eq. (13) to represent a fully self-consistent solution of the steady Euler equation, it is necessary to ensure that these line vortices are all steady under the effects of the local non-self-induced terms of the velocity field.

The parameters appearing in the conformal map Eq. (22) must be such that $z(\zeta)$ is a one-to-one mapping from the annulus $\rho<|\zeta|<1$ to the vortical region. It turns out that it is impossible to find any one-to-one conformal map of the form Eq. (22) from the annulus $\rho<|\zeta|<1$ unless $k \geqslant 3$ (the reasons for this will not be discussed here but are a result of certain mathematical properties of the class of domains to which we have restricted attention $\left.{ }^{24}\right)$. It has also been found that although $\rho$ necessarily lies in the range $0<\rho<1$, the map Eq. (22) is not one-to-one from the annulus $\rho<|\zeta|<1$ for all values of $\rho$ within this range. Indeed, for each $k$, there exists a maximum value of $\rho$ for which one-to-one maps of the form Eq. (22) exist. Moreover, the maximum value of $\rho$ for each $k$ (which we will denote by $\rho_{k}$ ) provides exactly the case of $k$ shielded Rankine vortices of the form Eq. (1) coming into contact and touching in an annular configuration about the origin. Thus the conformal map Eq. (22) and associated streamfunction Eq. (13) are precisely the required generalizations of the theory of Ref. 16 needed to answer the question posed in the introduction: what happens when multiple shielded Rankine vortices overlap?

\section{STEADINESS OF LINE VORTICES}

Define an auxiliary function $\hat{P}_{k}(\zeta)$ as follows:

$$
\hat{P}_{k}(\zeta)=\frac{P_{k}(\zeta)}{\left(1-\zeta^{k}\right)} \equiv \prod_{n=1}^{\infty}\left(1-\rho^{2 n k} \zeta^{k}\right)\left(1-\rho^{2 n k} \zeta^{-k}\right) \text {. }
$$

Now define

$$
F_{k}(\zeta)=\frac{P_{k}\left(\zeta^{-1} \eta_{1}^{-1}\right)}{\hat{P}_{k}\left(\zeta^{-1} \zeta_{1}^{-1}\right) \Pi_{j=1}^{k-1}\left(1-\zeta^{-1} \omega_{k}^{-j} \zeta_{1}^{-1}\right)} .
$$

Because line vortices move with the fluid, to ensure stationarity of the line vortices it is necessary to find the Laurent expansion (in the physical plane $z$ ) of $u-i v$ about each point $z_{j}$ at which a line vortex exists and ensure that all non-selfinduced contributions to the velocity field vanish at that point. This will mean that there is no net force on the line vortex-clearly a necessary condition for equilibrium. For the class of solutions under consideration, there is always a line vortex on the physical real axis at the (real) point $z_{1}$ $\equiv z\left(\zeta_{1}^{-1}\right)$. Some algebra reveals that at the point $z_{1}$ the condition that this line vortex is steady under the effects of the non-self-induced velocity terms is given by

$$
F_{k}^{\prime}\left(\zeta_{1}^{-1}\right)+F_{k}\left(\zeta_{1}^{-1}\right) \frac{z_{\zeta \zeta}\left(\zeta_{1}^{-1}\right)}{2 z_{\zeta}\left(\zeta_{1}^{-1}\right)}-\frac{1}{\zeta_{1}} \frac{P_{k}\left(\zeta_{1}^{-1} \eta_{1}^{-1}\right)}{P_{k}\left(\zeta_{1}^{-2}\right)}=0 .
$$

Equation (28) provides a relation between the parameters $\rho$ and $\zeta_{1}$ in the conformal map Eq. (22). Note that Eq. (28) is independent of the parameter $R$ which is essentially a normalization parameter.
It can be verified by straightforward algebra that, by the $k$-fold symmetry of the conformal map and the associated velocity field, Eq. (28) is also the condition for the remaining $k-1$ vortices at points $z\left(\zeta_{1}^{-1} \omega_{k}^{j}\right), j=1,2, . . k-1$ to be steady under the effects of the local non-self-induction terms. The parameter $R$ can be specified by specifying the total area of the vortical patch region.

If solutions for $\zeta_{1}, \rho$ and $\eta_{1}$ satisfying Eq. (23) and (28) which correspond to one-to-one conformal maps from the annulus $\rho<|\zeta|<1$ can be found then we have a solution which satisfies all the boundary conditions at both vortex patch boundaries, and ensured that the line vortices are steady under the effects of the non-self-induced velocity field.

It turns out that such solutions do indeed exist. For any integer $k \geqslant 3$ there exists a continuous one-parameter family of solutions parametrized by the conformal modulus $\rho$. Once $\rho$ is specified, $\zeta_{1}$ and $\eta_{1}$ follow from the two conditions Eqs. (28) and (23), while $R$ then follows from a specification of the total area of the vortical patch region.

The critical $\rho=\rho_{k}$ corresponds to a situation in which zeros of the conformal mapping function $z_{\zeta}$ approach the boundary of the annulus $\rho<|\zeta|<1$ from outside (recall that for conformality, a necessary condition is that all such zeros lie outside this annulus). In fact, the zeros approach this annulus along the rays $\arg [\zeta]=\mu_{k}$ where $\mu_{k}$ are the $k$-th roots of -1 . The equation providing the critical value of $\rho=\rho_{k}$ can be found by simultaneously solving Eqs. (28), (23) as well as the additional equation

$$
z_{\zeta}\left(e^{i \pi / k}\right)=0
$$

for the three (critical) parameters $\zeta_{1}, \eta_{1}$, and $\rho$. Note that the argument of $z_{\zeta}$ in Eq. (29) is simply the root of -1 lying in the first quadrant. These critical solutions correspond to a situation in which $k$ shielded Rankine vortices just come into contact in a $k$-symmetric annular configuration. The value of $\rho$ obtained will be the maximum $\rho=\rho_{k}$ for which solutions of this kind exist, i.e., for each $k \geqslant 3$, solutions exist for all $\rho$ satisfying

$$
0 \leqslant \rho \leqslant \rho_{k} .
$$

The first few values of $\rho_{k}$ are found numerically, to three decimal places, to be given by

$$
\rho_{3}=0.567, \quad \rho_{4}=0.671, \quad \rho_{5}=0.732, \quad \rho_{6}=0.799 \text {. }
$$

Summary of the exact solutions. For convenience, we now summarize the important formulas. It has been shown that a class of exact solutions for an annular multipolar array of $k$ vortices is described by a conformal map from the annulus $\rho<|\zeta|<1$ given by Eq. (22) for any integer $k \geqslant 3$ with real parameters $\rho, \eta_{1}, \zeta_{1}$ and $R$ related via Eq. (23) and existing in the ranges

$$
0 \leqslant \rho \leqslant \rho_{k}, \quad 1<\zeta_{1}<\rho^{-1}, \quad 1<\eta_{1}<\rho^{-1},
$$

and also satisfying the nonlinear algebraic constraint Eq. (28). Using the fact that 


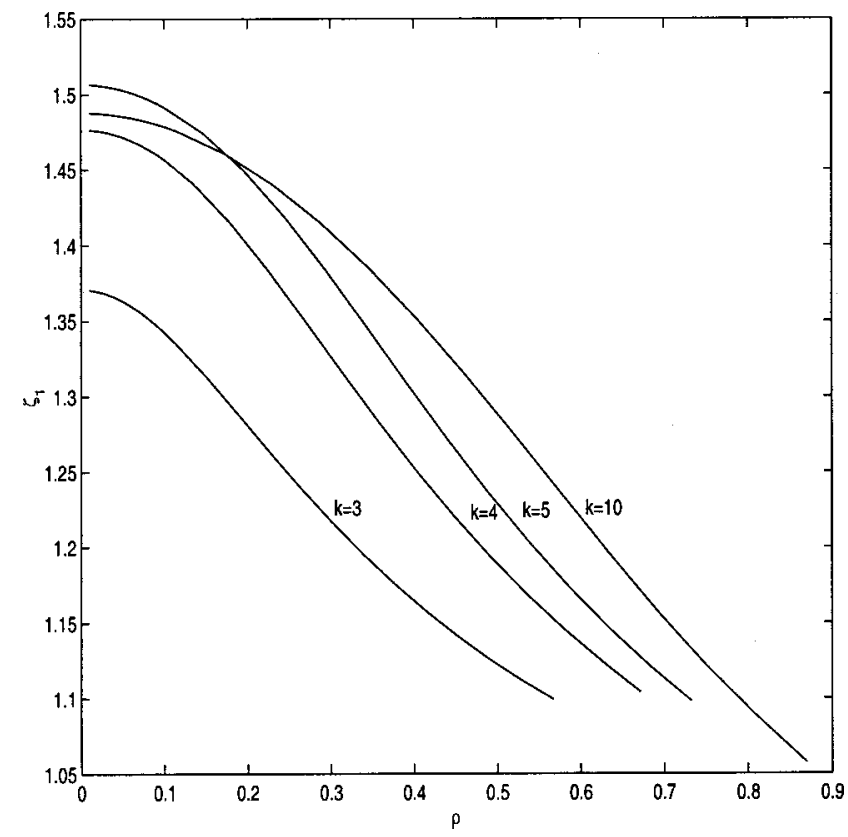

FIG. 3. Graph of the solution for $\zeta_{1}(y$-axis) of the stationarity condition Eq. (28) against $\rho$ (x-axis) for $k=3,4,5$, and 10 .

$$
2 i \psi_{z}=2 i \frac{\partial \psi}{\partial z}=i \frac{\partial \psi}{\partial x}+\frac{\partial \psi}{\partial y}=u-i v
$$

the associated velocity field inside $D$ is given (as a function of $\zeta$ and $\bar{\zeta})$ as

$u-i v=2 i \psi_{z}=-\frac{i \omega R}{2}\left(\bar{\zeta} \frac{P_{k}\left(\bar{\zeta} \eta_{1}^{-1}\right)}{P_{k}\left(\bar{\zeta} \zeta_{1}^{-1}\right)}-\frac{1}{\zeta} \frac{P_{k}\left(\zeta^{-1} \eta_{1}^{-1}\right)}{P_{k}\left(\zeta^{-1} \zeta_{1}^{-1}\right)}\right)$.

These solutions represent a continuous one-parameter family of vortices for each integer $k \geqslant 3$. We can choose $\rho$ to be the relevant governing geometrical parameter. The parameter $R$ is arbitrary but one choice is to specify the total area of the vortical patch to be, say, $\pi$, i.e.,

$\pi=\frac{1}{2} \operatorname{Im}\left[\oint_{|\zeta|=1} \bar{z}\left(\zeta^{-1}\right) z_{\zeta}(\zeta) d \zeta-\oint_{|\zeta|=\rho} \bar{z}\left(\zeta^{-1}\right) z_{\zeta}(\zeta) d \zeta\right]$

where we have used Eq. (19). To find the critical $\rho_{k}$, we simultaneously solve Eqs. (23), (28), and the equation

$$
z_{\zeta}\left(e^{\pi i / k}\right)=0
$$

for $\zeta_{1}, \eta_{1}$, and $\rho_{k}$.

\section{DISCUSSION OF THE SOLUTIONS}

In Fig. 3, the graphs of $\zeta_{1}$ against $\rho$ as determined by solving Eqs. (23) and (28) using a numerical Newton iterative procedure are shown for $k=3,4,5$, and 10 for $\rho$-values within the respective range of existence of solutions in each case. Given $k$ and $\rho$, the corresponding $\zeta_{1}$ is given by these graphs. The value of $\eta_{1}$ then follows from Eq. (23). $R$ follows from the area normalization condition once $\rho, \zeta_{1}$ and $\eta_{1}$ are determined.
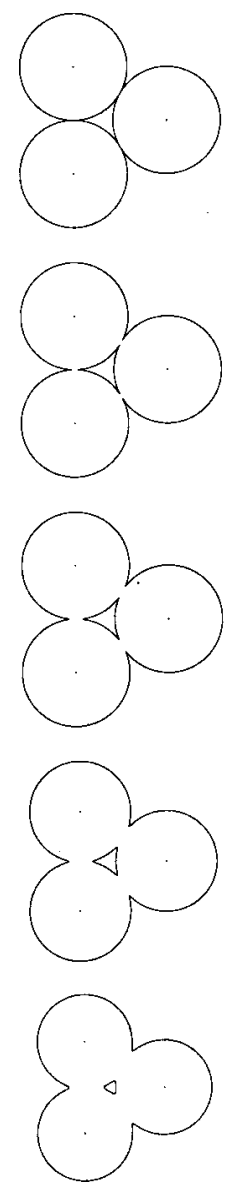

FIG. 4. Vortex boundaries and line vortex positions (shown as dots): $k$ $=3 ; \rho=0.56,0.4,0.3,0.2,0.1$.

We first investigate the shapes of the vortices as the geometrical parameter $\rho$ is varied. Note that, although the conformal map Eq. (22) is represented as an infinite product, because the conformal modulus $\rho$ is less than 1 , the vortex shapes can be plotted by truncating the infinite products Eq. (20) after a suitably large number of terms. A convenient test of whether enough terms have been retained is provided by checking that the functional relation Eq. (19) is well satisfied for arbitrary choices of $\zeta$. In practice, it is found that only a few terms need to be retained.

In Fig. 4, the shapes of the vortical regions for various values of $\rho$ are plotted for a class of vortices with $k=3$. The positions of the line vortices are also indicated by dots within the patch. The first plot is a near-critical case with $\rho=0.56$. It can be seen that this corresponds to a situation in which three shielded Rankine vortices just come into contact forming a doubly connected annular region of vorticity enclosing a region of irrotational flow. As $\rho$ decreases, the three initial shielded Rankine vortices merge to form a compound vortical structure. It is interesting to note that a domain of this particular kind was first constructed, using very different methods, by Gustafsson, ${ }^{29}$ who was interested in purely mathematical problems in quadrature domain theory. Here we have shown the applicability of these abstract mathematical results to finding physical solutions of the Euler equation. 

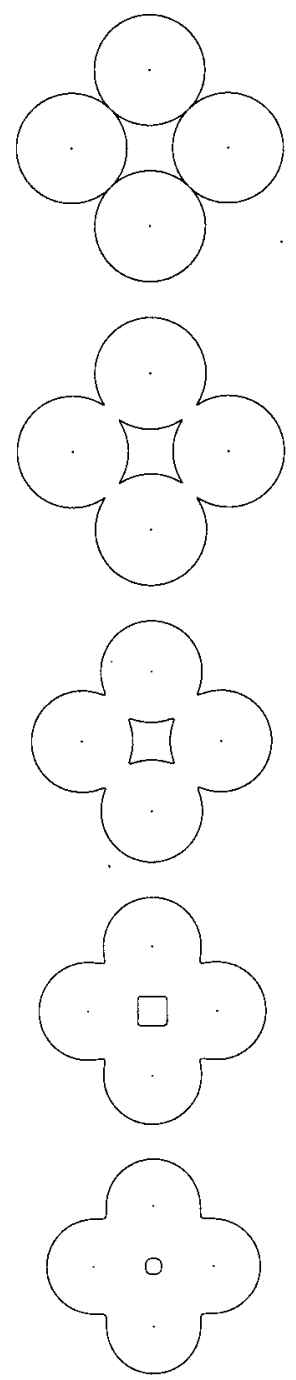

FIG. 5. Vortex boundaries and line vortex positions (shown as dots): $k$ $=4 ; \rho=0.65,0.4,0.3,0.2,0.1$.

In Fig. 5, the analogous shapes are given for a class of vortices with $k=4$, while Figs. 6 and 7 give the cases $k=5$ and $k=10$, respectively. In each case, the first plot corresponds to a near-critical value of $\rho$ so that the configurations resemble an annular configuration of touching shielded Rankine vortices. As $\rho$ decreases, the size of the enclosed irrotational region decreases until it eventually disappears when $\rho=0$ resulting in a simply connected region of vorticity.

The shapes of these compound vortices have many similarities with the shapes computed numerically by Dritschel ${ }^{20}$ in his calculations of the breakup of a uniform vortical annulus into multiple vortices. In particular, it is interesting to compare the vortical shapes in Figs. 4-7 here (especially those corresponding to smaller $\rho$ values) to some of those featured in Figs. 3-6 and 10 of Dritschel. ${ }^{20}$

Figures 8-10 show typical streamlines and circulation regions associated with the merged compound vortices. To plot the streamlines, it is found most convenient to use Eq. (34) to compute the components $(u, v)$ of the velocity field for a discretized set of points inside the unit disc in the $\zeta$-plane. These data are then fed into a data visualization
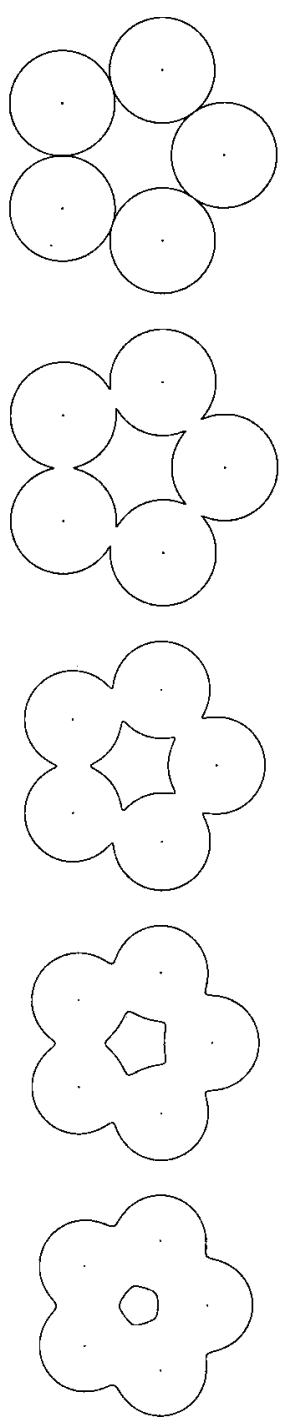

FIG. 6. Vortex boundaries and line vortex positions (shown as dots): $k$ $=5 ; \rho=0.72,0.5,0.4,0.3,0.2$.

package which integrates an interpolated velocity field for the streamlines numerically. This procedure gives very good results in general. We point out, however, that certain streamlines that draw too close to the stagnation points are sometimes found not to "close" completely due to inaccuracies in the numerical integration. Also, streamlines too close to the boundaries of the patch (where the magnitude of the velocity field gets small) are similarly hard to calculate accurately in this way. These inaccuracies are purely numerical-in theory, these streamlines close. While in principle it is possible to use the explicit expression for the Schwarz function to integrate the expression Eq. (13) analytically for the streamfunction, there seems to be no simple closed form expression for the resulting primitives. The streamfunction could therefore be represented as an explicit set of integrals, however, to actually plot the streamlines would still require numerical quadrature of these integrals.

In Fig. 8, streamlines of the principal circulation regions of a typical $k=4$ vortex are shown, while Figs. 9 and 10 depict the circulation regions of a typical multipolar vortex with $k=5$ and $k=10$, respectively. The streamlines shown in 

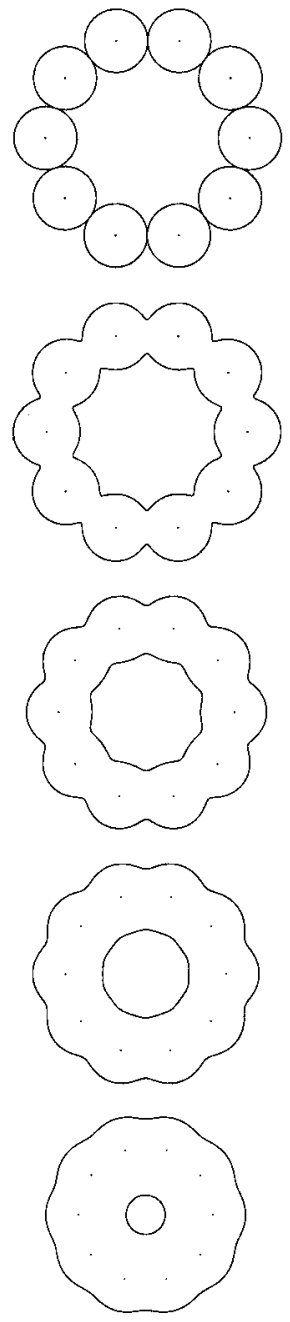

FIG. 7. Vortex boundaries and line vortex positions (shown as dots): $k$ $=10 ; \rho=0.85,0.6,0.5,0.4,0.2$.

bold are the inner and outer boundaries of the vortical region. Typically, one observes that there exists $k$ stagnation points at which separatrix streamlines converge marking out distinct regions of recirculation. Due to difficulties in plotting streamlines through the stagnation points, none of the separatrix streamlines are shown in Figs. 8 and 9, but streamlines drawing close to the separatrix streamlines are featured in Fig. 10 in the case $k=10$. When $\rho$ is close to (but just below) the critical $\rho_{k}$ for each $k$, the area occupied by streamlines which circulate around the entire structure (as opposed to circulating locally around one of the satellites) is small, as is the area of the streamlines which circulate around the enclosed region of irrotational fluid. These areas increase as $\rho$ decreases toward zero, leading to a greater area of streamlines circulating around the entire vortical structure and therefore to greater global interaction between the separate shielded vortices which have merged to form the compound structure.

The strength, $\Gamma_{k}$ say, of each of the $k$ line vortices superposed on the otherwise uniform patch of vorticity is

$$
\Gamma_{k}=-\frac{\omega \pi}{k},
$$

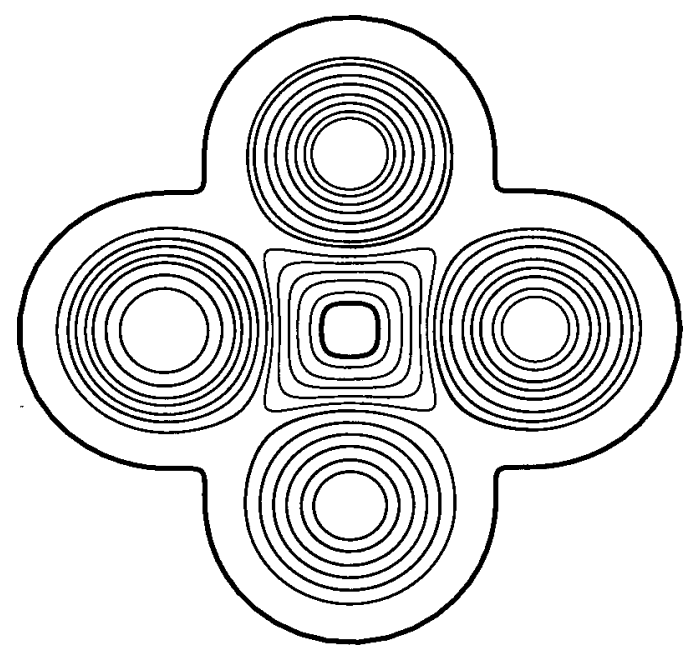

FIG. 8. Typical streamlines $k=4 \quad\left(\rho=0.118\right.$ and $\left.\zeta_{1}=1.453\right)$ : the bold streamlines are the inner and outer boundaries of the vortex patch.

regardless of the value of $\rho$ or $\zeta_{1}$. This result is clear: the total circulation of the structure is zero while the vorticity associated with the uniform patch, of total area $\pi$, is $\omega$. The strengths of the line vortices must therefore be given by Eq. (37) if the combined structure is to have zero total circulation. One can ask the following hypothetical question: in how many geometrical configurations can one arrange a uniform patch of vorticity of area $\pi$ and strength $\omega$ with $k$ line vortices each of strength $-(\omega \pi / k)$ such that the combined structure is a global equilibrium solution of the Euler equation? The above results reveal that there is at least a continuous one parameter family (parametrized here by $\rho$ ) of ways to do this and therefore a dense set of such multipolar configurations. The apparently dense nature of the class of mul-

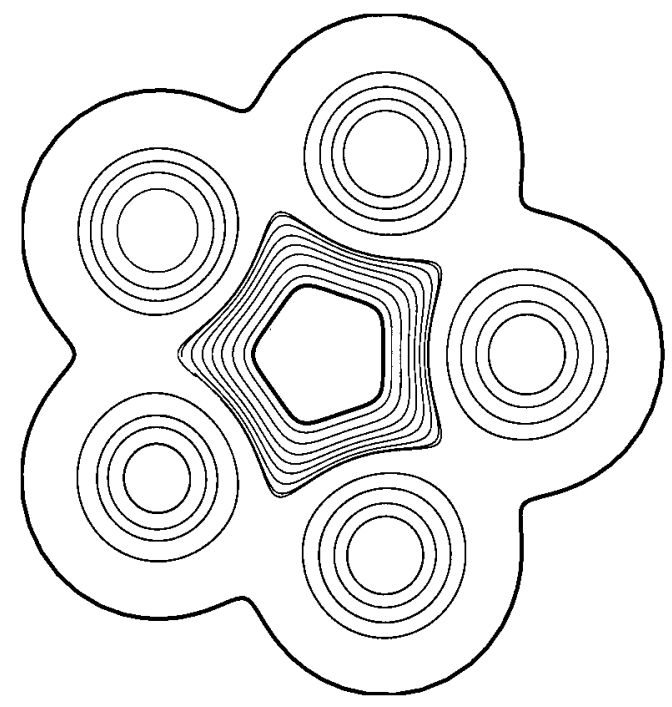

FIG. 9. Typical streamlines $k=5 \quad\left(\rho=0.227\right.$ and $\left.\zeta_{1}=1.436\right)$ : the bold streamlines are the inner and outer boundaries of the vortex patch. 


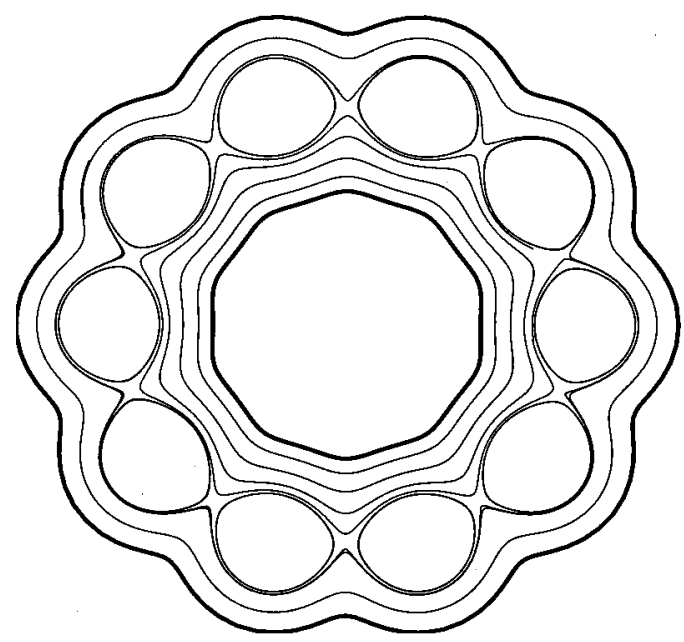

FIG. 10. Typical streamlines $k=10\left(\rho=0.4327\right.$ and $\left.\zeta_{1}=1.333\right)$ : the bold streamlines are the inner and outer boundaries of the vortex patch.

tipolar equilibrium solutions of the Euler equation has been pointed out before ${ }^{30}$ and we comment further on this point in the conclusion.

As mentioned earlier, the limit $\rho \longrightarrow 0$ gives a simply connected vortical region which has $k$ satellite vortical regions and a central core region (enclosed by the separatrix streamlines) of uniform vorticity. In fact, as recently discussed in Crowdy and Cloke, ${ }^{31}$ this simply connected patch solution is contained within the class of solutions ${ }^{16}$ and can be found by using the methods of Ref. 16 and considering conformal maps from the unit $\zeta$-circle which have the rational function form

$$
z(\zeta)=\frac{R(a) \zeta}{\zeta^{k}-a^{k}} .
$$

Note that this form is different to that considered in Ref. 16. Only one single value of the parameter $a$ in Eq. (38) will satisfy the associated stationarity condition of the line vortices in this case and therefore give an exact solution of the steady Euler equation. The new solutions presented here can therefore also be understood as a continuation of the simply connected solutions Eq. (38) into nonzero $\rho$ (corresponding physically to the formation of an enclosed central region of irrotational fluid).

A topic of much recent research concerns the stability of multipolar vortex structures. Usually, such stability questions are examined numerically and stability studies often involve first solving numerically for a multipolar equilibrium solution and then perturbing it (numerically) in some way. The class of solutions found both here and in Ref. 16 not only have the advantage of being describable by means of exact, closed-form formulas but also have advantages when it comes to studying their stability properties. First, the linear stability matrices associated with small (linear) perturbations can be written down in closed form from exact knowledge of the base state solution being perturbed. Second, because the exact solutions consist of a combination of two of the most well-studied idealizations of two-dimensional vortex patches, i.e., line vortices and uniform vortex patches, their nonlinear stability properties can be studied using simple adaptations of existing numerical contour dynamics codes.

The linear and nonlinear stability properties of the multipolar solutions presented in Ref. 16 has recently been studied in Ref. 31. Many of the stability properties of the exact solution class are found to be consistent with previous stability studies of multipolar vortices. The recent results of Crowdy and Cloke ${ }^{31}$ suggest that there is a good possibility that the solutions found here might be robust structures, if not actually linearly/neutrally stable. This is because the solutions here resemble certain of the multipolar solutions of Crowdy $^{16}$ in the case where the satellite line vortices are well-separated and the central line vortex is comparatively weak (the solutions here, of course, possess no central line vortex but the overall qualitative distribution of vorticity is similar). Recent results ${ }^{31}$ reveal that such configurations are neutrally stable in the case $k=3$ while the cases $k=4$ and $k=5$ are neutrally stable provided the satellite line vortices are sufficiently separated and, under moderate nonlinear perturbation, prove relatively robust. A detailed study would be required to determine whether the solutions found here inherit these linear stability traits. The linear stability properties of a concentric annular vortex of uniform unit vorticity as considered by Michalke and Timme ${ }^{32}$ have been found to depend crucially on the conformal modulus $\rho$ of the annulus. Indeed, the annulus becomes linearly stable as the conformal modulus decreases below the critical value of $\rho=\frac{1}{2}$. We conjecture that the linear stability properties of the new annular multipolar structures will similarly have an important dependence on $\rho$. The dependence of the linear stability on the symmetry parameter $k$ is also of interest.

\section{CONCLUSION}

A new class of nontrivial exact solutions of the Euler equation possessing regions of distributed vorticity reminiscent of the nonlinear multipolar saturation states of an unstable uniform vortical annulus (cf. Dritschel ${ }^{20}$ ) has been derived.

It has been shown how mathematical properties of the Schwarz function ${ }^{21}$ combine with the physical constraints relevant to steady vortical flows to produce classes of multipolar equilibria of the Euler equations. The solutions presented here and in Crowdy ${ }^{16}$ reveal that the modified Schwarz potential Eq. (8) provides a natural mathematical device for understanding how a finite-area patch of uniform vorticity interacts with a finite set of superposed line vortices to form global equilbria of the Euler equation. Furthermore, Crowdy ${ }^{33}$ has recently extended the same mathematical ideas to derive a class of exact solutions involving rotating vortex arrays with distributed vorticity which generalize Thomson's classic study of co-rotating line vortex configurations. ${ }^{23}$

Any two shielded Rankine vortices only interact when they overlap and it has been shown that there exists a nonlinear superposition property which means that one can construct global multipolar equilibria of the Euler equation with an annular doubly connected distribution of nonzero vorticity by merging these invisible vortical structures in the manner described herein. This suggests that it might be possible to 
construct multipolar equilibria having vortical regions of even more complicated topological structure, and thereby move toward a general nonlinear superposition method for vortex equilibria. Further work in this direction has been carried out in Crowdy ${ }^{26}$ where a general constructive method is formulated based on ideas from quadrature domain theory. $^{21,24}$

The geometry/topology of vortex equilibria is of great interest. Campbell and Ziff ${ }^{34}$ have catalogued stable and unstable equilibria of $N$ line vortices for up to $N=50$. With reference to this study, Pullin ${ }^{30}$ suggests that "if all such equilibria can be continued to finite and perhaps different area and uniform vorticity for each vortex then the class of finite area $N$-vortex equilibria in expected to be very dense, excluding even the possibility of further bifurcations off each branch for given $N$...the class of multiple-vortex equilbria is very large." We believe that our results, suggestive as they are of a general nonlinear superposition principle for vortex equilibria, provide evidence to corroborate this statement.

Finally, a note on the physical detection of these vortices using remote measurements. The "invisibility" of the solutions found both here and in Ref. 16 means that their presence cannot be detected by any remote measurements of the induced far-field velocity. Moreover, the results here show that there is a high degree of nonuniqueness in the class of localized vortical structures possessing this invisibility property. An interesting and physically important question is: how does one ascertain the structure of these vortices by means of remote measurements? Such questions of remote detection arise in various physical contexts such as oceanic data assimilation. In practice, three-dimensional effects will presumably enter, however, it is interesting that there exist large classes of geometrically distinct two-dimensional vortices whose far-field moments vanish identically at all orders.

\section{ACKNOWLEDGMENTS}

The author gratefully acknowledges financial support from the National Science Foundation (Grant No. DMS9803167 and DMS-9803358) as well as financial support from the Nuffield Foundation.

${ }^{1}$ J. C. McWilliams, "The emergence of isolated coherent vortices in turbulent flow," J. Fluid Mech. 146, 21 (1984).

${ }^{2}$ B. Legras, P. Santangelo, and R. Benzi, "High resolution numerical experiments for forced two dimensional turbulence," Europhys. Lett. 5, 37 (1988).

${ }^{3}$ G. J. F. van Heijst and R. Kloosterziel, "Tripolar vortices in a rotating fluid," Nature (London) 338, 569 (1989).

${ }^{4}$ G. J. F. van Heijst, R. Kloosterziel, and C. W. M. Williams, "Laboratory experiments on the tripolar vortex in a rotating fluid," J. Fluid Mech. 225, 301 (1991)

${ }^{5}$ M. Beckers and G. J. F. van Heijst, "The observation of a triangular vortex in a rotating fluid," Fluid Dyn. Res. 22, 265 (1998).
${ }^{6}$ G. F. Carnevale and R. C. Kloosterziel, "Emergence and evolution of triangular vortices," J. Fluid Mech. 259, 305 (1994).

${ }^{7}$ P. Orlandi and G. J. F. van Heijst, "Numerical simulations of tripolar vortices in 2D flow," Fluid Dyn. Res. 9, 1147 (1992).

${ }^{8} \mathrm{X}$. J. Carton and B. Legras, "The life-cycle of tripoles in two-dimensional incompressible flows," J. Fluid Mech. 267, 53 (1992).

${ }^{9}$ X. J. Carton, G. R. Flierl, and L. M. Polvani, "The generation of tripoles from unstable axisymmetric isolated vortex structures," Europhys. Lett. 9, 339 (1989).

${ }^{10}$ L. M. Polvani and X. J. Carton, "The tripole: A new coherent vortex structure of incompressible two-dimensional flows," Geophys. Astrophys. Fluid Dyn. 51, 87 (1990).

${ }^{11}$ Y. G. Morel and X. J. Carton, "Multipolar vortices in two dimensional incompressible flows," J. Fluid Mech. 267, 23 (1994).

${ }^{12}$ R. D. Pingree and B. LeCann, "Three anticyclonic slope water oceanic eddies (swoddies) in the southern Bay of Biscay," Deep-Sea Res. 39, 1147 (1992).

${ }^{13}$ C. E. Leith, "Minimum enstrophy vortices," Phys. Fluids 27, 1388 (1984).

${ }^{14}$ O. U. Velasco Fuentes, G. J. F. Van Heijst, and N. P. M. Van Lipzig, "Unsteady behaviour of a topography-modulated tripole," J. Fluid Mech. 307, 11 (1996).

${ }^{15}$ R. C. Kloosterziel and G. F. Carnevale, "On the evolution and saturation of instabilities of two-dimensional isolated circular vortices," J. Fluid Mech. 388, 217 (1999).

${ }^{16}$ D. G. Crowdy, "A class of exact multipolar vortices," Phys. Fluids 11, 2556 (1999).

${ }^{17}$ P. G. Saffman and R. Szeto, "Equilibrium shapes of a pair of equal uniform vortices," Phys. Fluids 23, 2339 (1980).

${ }^{18}$ R. T. Pierrehumbert, "A family of steady, translating vortex pairs with distributed vorticity,” J. Fluid Mech. 99, 129 (1980).

${ }^{19}$ D. G. Dritschel, "The stability and energetics of co-rotating uniform vortices," J. Fluid Mech. 157, 95 (1985).

${ }^{20}$ D. G. Dritschel, "The nonlinear evolution of rotating configurations of uniform vorticity," J. Fluid Mech. 172, 157 (1986).

${ }^{21}$ P. J. Davis, "The Schwarz function and its applications," in Carus Mathematical Monographs (The Mathematical Association of America, Washington, D.C., 1974).

${ }^{22}$ E. A. Overman, "Steady-state solutions of the Euler equations in two dimensions: Local analysis of limiting V-states," SIAM (Soc. Ind. Appl. Math.) J. Appl. Math. 46, 765 (1986).

${ }^{23}$ J. J. Thomson, " On the Motion of Vortex Rings (Adams Prize essay 1882) (MacMillan, London, 1883).

${ }^{24}$ H. S. Shapiro, The Schwarz Function and its Generalization to Higher Dimensions (Wiley, New York, 1992).

${ }^{25}$ P. G. Saffman, Vortex Dynamics (Cambridge University Press, Cambridge, 1992).

${ }^{26}$ D. G. Crowdy, "Multipolar vortices and algebraic curves," Proc. R. Soc. London, Ser. A 457, 2337 (2001).

${ }^{27}$ Z. Nehari, Conformal Mapping (McGraw-Hill, New York, 1952).

${ }^{28} \mathrm{G}$. Valiron, Cours d'analyse Mathematique, Theorie des Fonctions, 2nd ed. (Masson et Cie, Paris, 1947).

${ }^{29} \mathrm{~B}$. Gustafsson, "Singular and special points on quadrature domains from an algebraic geometric point of view," J. Analyse Math. 51, 91 (1988).

${ }^{30}$ D. I. Pullin, "Contour dynamics methods," Annu. Rev. Fluid Mech. 24, 89 (1992).

${ }^{31}$ D. G. Crowdy and M. Cloke, "Stability analysis of a class of twodimensional multipolar vortex equilibria," Phys. Fluids (submitted).

${ }^{32} \mathrm{~A}$. Michalke and A. Timme, "On the inviscid instability of certain twodimensional vortex-type flows," J. Fluid Mech. 29, 647 (1967).

${ }^{33}$ D. G. Crowdy, "Exact solutions for rotating vortex arrays with finite-area cores," J. Fluid Mech. (submitted).

${ }^{34}$ L. J. Campbell and R. M. Ziff, "A catalogue of two-dimensional vortex patterns,” Los Alamos Rep. LA-7384-MS, 1978. 